\title{
Inferior Frontal Gyrus
}

National Cancer Institute

\section{Source}

National Cancer Institute. Inferior Frontal Gyrus. NCI Thesaurus. Code C32774.

A region on the surface of the frontal lobe visibly divided into three parts: the pars

opercularis, the pars triangularis, and the pars orbitalis. It is bound by the inferior frontal sulcus dorsally and the lateral fissure ventrally. 